\title{
Time-resolved magnetic resonance angiography and flow-sensitive 4-dimensional magnetic resonance imaging at 3 Tesla for blood flow and wall shear stress analysis
}

\author{
Alex Frydrychowicz, MD, ${ }^{a}$ Alexander Berger, ${ }^{a}$ Maximilan F. Russe, ${ }^{a}$ Aurélien F. Stalder, MS, ${ }^{a}$ Andreas Harloff, MD, \\ Sven Dittrich, MD, ${ }^{\mathrm{c}}$ Jürgen Hennig, $\mathrm{PhD},{ }^{\mathrm{a}}$ Mathias Langer, MD, MBA, ${ }^{\mathrm{a}}$ and Michael Markl, $\mathrm{PhD}^{\mathrm{a}}$
}

From the Department of Diagnostic Radiology and Medical Physics, ${ }^{\text {a }}$ Department of Neurology and Clinical Neurophysiology, ${ }^{b}$ and Department of Pediatric Cardiology, University Hospital Freiburg, Freiburg, Germany.

The authors have no disclosures. Dr Markl receives funding from Deutsche Forschungsgemeinschaft Grant MA 2383/3-1.

Received for publication Aug 23, 2007; revisions received Dec 26, 2007; revised May 2008; accepted for publication Feb $19,2008$.

Address for reprints: Alex Frydrychowicz, MD, University Hospital Freiburg, Department of Diagnostic Radiology, Hugstetter Str. 55, 79106 Freiburg, Germany (E-mail: alex.frydrychowicz@uniklinik-freiburg.de).

J Thorac Cardiovasc Surg 2008;136:400-7

$0022-5223 / \$ 34.00$

Copyright $(\underset{2008}{ } 20 y$ The American Association for Thoracic Surgery

doi:10.1016/j.jtcvs.2008.02.062
Objectives: In light of the ongoing discussion about flow-mediated arterial remodeling, it was the aim of this report to demonstrate the detailed assessment of 3-dimensional vascular hemodynamics by high-field magnetic resonance imaging in healthy volunteers and to illustrate its potential in comparison with results in a patient with stenosis.

Materials and Methods: All examinations consisted of flow-sensitive 4-dimensional magnetic resonance imaging at 3 Tesla. Retrospective blood flow visualization and segmental quantification of wall shear stress and oscillatory shear index were performed. The results from 11 healthy individuals were compared with a 13-year-old patient with aortic stenosis who received a combined protocol with time-resolved 3-dimensional magnetic resonance angiography before and 5 and 9 months after intervention.

Results: Evaluation of normal blood flow characteristics demonstrated predominantly right-handed helical flow in the ascending aorta. Vortex formation was observed in 1 of 11 volunteers. Consistently high segmental wall shear stress was found along the circumference of the ascending aorta (average wall shear stress $=$ $0.191 \pm 0.06 \mathrm{~N} / \mathrm{m}^{2}$ ) and descending aorta (average $0.191 \pm 0.06 \mathrm{~N} / \mathrm{m}^{2}$ ). Compared with volunteers, the patient revealed substantial flow changes proximal and distal to the stenosis. Blood flow alterations in the ascending aorta were also observed associated with changes in velocities and wall shear stress that gradually normalized after intervention.

Conclusion: Flow-sensitive 4-dimensional magnetic resonance imaging at 3 Tesla can provide deeper insights into hemodynamic alterations in the diagnosis and follow-up of aortic pathologies. These findings indicate the potential of the methodology for the evaluation of effects of localized pathologies on the entire vascular system, which will have to be confirmed in future studies.

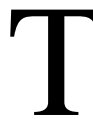
The impact of hemodynamic alterations on vessel wall remodeling has gained attention during the past 20 years, and noninvasive methods for the detailed characterization of such changes have recently been adapted for in vivo diagnostics. ${ }^{1-3}$ The application of derived parameters such as wall shear stress (WSS) to monitor therapy or find parameters predictive for progression or relapse of an arterial disease could enrich modern diagnostic and therapeutic decision making. In diseases such as aortic stenosis, patients are at a high risk of secondary complications even after successful therapy. Among other concerns, the formation of aneurysms has potentially serious complications. However, the mechanisms associated with aneurysm formation either at the site of stenosis or in the ascending aorta (AAo) ${ }^{4}$ are not completely understood, and the association with altered hemodynamics is still hypothetical. 


$$
\begin{array}{ll}
\text { Abbreviations and Acronyms } \\
\begin{array}{ll}
\text { 3D } & =3 \text { dimensional } \\
\text { 4D } & =4 \text { dimensional } \\
\text { AAo } & =\text { ascending aorta } \\
\text { CE-MRA } & =\text { contrast-enhanced magnetic resonance } \\
& \text { angiography } \\
\text { DAo } & =\text { descending aorta } \\
\text { MRI } & =\text { magnetic resonance imaging } \\
\text { OSI } & =\text { oscillatory shear index } \\
\text { T } & =\text { Tesla } \\
\text { WSS } & =\text { wall shear stress }
\end{array}
\end{array}
$$

Because of its beneficial effects on signal-to-noise ratio, ${ }^{5}$ clinical high-field magnetic resonance imaging (MRI) has the potential to enhance the depiction of arterial pathologies and associated functional changes. The combination of methodologically advanced imaging strategies, such as view sharing, ${ }^{6}$ parallel imaging, ${ }^{7}$ and partial Fourier transform, ${ }^{8}$ with fast and functional imaging is currently possible. Thus, high-field imaging at 3 Tesla (T) may contribute to a better understanding of the interaction of alterations in vascular geometry and changes in blood flow and vessel wall characteristics. Previous reports show that the combination of morphologic and functional vascular MRI can reveal comprehensive findings in vascular geometry under physiologic conditions ${ }^{9}$ and in the presence of pathologic alterations in vascular geometry. ${ }^{3,10}$ To further underline the additional information provided by modern time-resolved contrast-enhanced magnetic resonance angiography (CE-MRA) ${ }^{11,12}$ and flow-sensitive 4-dimensional MRI, ${ }^{13}$ we compare the collective findings of 11 healthy volunteers with the blood flow and derived vessel wall characteristics in a 13 -year-old boy with high-grade descending aortic stenosis. We provide detailed information about the changes in vascular hemodynamics 5 and 9 months after intervention (ie, stent placement and dilatation of the stenosis).

\section{Materials and Methods}

Flow experiments were performed in 11 healthy volunteers $(22.6 \pm$ 1.4 years, $67.7 \pm 9.0 \mathrm{~kg}, 2$ female, 9 male) and a 13 -year-old patient before and 5 and 9 months after stent placement and dilatation of a high-grade aortic stenosis. Studies were approved by the local ethics committee and performed after written informed consent on a 3 T magnetic resonance system (Magnetom TRIO, Siemens, Germany, maximum gradient strength $=40 \mathrm{mT} / \mathrm{m}$, rise time $=200$ $\mu$ sec, 8-channel receiver coil) using an rf-spoiled gradient echo sequence with interleaved 3-directional velocity encoding during free breathing and prospective electrocardiogram gating. Data were acquired with $\alpha=15^{\circ}$, venc $=150 \mathrm{~cm} / \mathrm{s}$, spatial resolution $(3.2 \times$ $2.1 \times 5.0 \mathrm{~mm}^{3}$ flip angle $(\mathrm{FA})$, echo time $(\mathrm{TE})=3.5 \mathrm{~ms}$, repetition time $(\mathrm{TR})=5.6 \mathrm{~ms}$, and temporal resolution $=49 \mathrm{~ms}$. To minimize breathing artifacts and image blurring, respiration control was per- formed on the basis of combined adaptive k-space reordering and navigator gating. ${ }^{14}$

Blood flow quantification and WSS analysis were performed using a homebuilt software tool based on MatLab (The MathWorks Inc, Natick, Mass) that allowed for vessel lumen segmentation of 2-dimensional cutplanes. Freely positioned cutplanes were extracted from the 4-dimensional flow data using a commercially available software package (EnSight, CEI, Apex, NC) that also served to generate time-resolved, color-encoded visualization of flow characteristics. ${ }^{15}$ Further visual evaluation of datasets was performed by 2 experienced readers in a consensus reading screening for general hemodynamics, blood flow helicity, development, size and direction of vortices, and degree of physiologic retrograde flow.

For time-resolved 3-dimensional (3D) MRA, an rf-spoiled gradient echo sequence with parallel imaging ( $k$-space based GRAPPA reconstruction ${ }^{7}$ ) with an acceleration factor of 4 along the phase encoding direction and 32 reference lines, partial Fourier acquisition along the phase and slice encoding direction (for both, partial Fourier factor $=6 / 8$ ), and view sharing along the temporal domain (TREAT imaging $^{6}$ ) based on elliptical centric view ordering was used. With the resulting imaging protocol, $20 \mathrm{~T} 1 \mathrm{w} 3 \mathrm{D}$ data volumes were acquired consecutively with a temporal update rate of $2.8 \mathrm{sec}-$ onds. CE-MRA was performed using gadobenate dimeglumine (Gd-BOPTA chelate, Multihance, Altana Pharma, Konstanz, Germany, molar concentration $0.5 \mathrm{~mol} / \mathrm{L}$, single dose $=0.1 \mathrm{mmol} / \mathrm{kg}$ body weight, injection rate $=3 \mathrm{~mL} / \mathrm{s}$ ). Further imaging parameters were as follows: flip angle $(\mathrm{FA})=14$ degrees, matrix $=224 \times 320$, field of view $280 \times 400 \mathrm{~mm}^{2}, \mathrm{TR}=2.12 \mathrm{~ms}$, TE $=0.85 \mathrm{~ms}$, slice thickness $=1.25 \mathrm{~mm}$, effective spatial resolution $=1.96 \times 1.25 \times$ $1.25 \mathrm{~mm}^{3}$, and temporal update rate $=2.82$ seconds.

\section{Results}

\section{Normal Aortic Hemodynamics and Wall Parameters}

Flow-sensitized 4-dimensional (4D) MRI covering the entire thoracic aorta was successfully performed in all healthy volunteers. Subsequent visualization of the measured blood flow velocities and directions included 3D streamline depiction of flow patterns. Figure 1 illustrates the potential of 3D blood flow visualization to analyze global and regional hemodynamics in the thoracic aorta. In healthy subjects, predominantly right-handed helical flow was seen in the AAo and vortices occurred only occasionally (1/11 volunteers). The results of visual 3D flow grading for all 11 volunteers (mean diameter of the AAo $=2.4 \pm 0.2 \mathrm{~cm}$ ) are summarized in Table 1.

With respect to the ongoing discussion of the importance of WSS and its implications for vascular remodeling, ${ }^{1}$ a quantification of WSS and flow was conducted in the AAo and descending aorta (DAo) by retrospectively extracting manually positioned 2-dimensional cutplanes from the 3D dataset. Next, segmentation of the aortic wall was interactively performed frame by frame using cubic B-spline smooth contours. Axial WSS was directly derived from the measured 3-directional velocity vector field using a deformation tensor. ${ }^{16}$ The oscillatory shear index (OSI) was calculated as the ratio between the area under the WSS time curve of the 

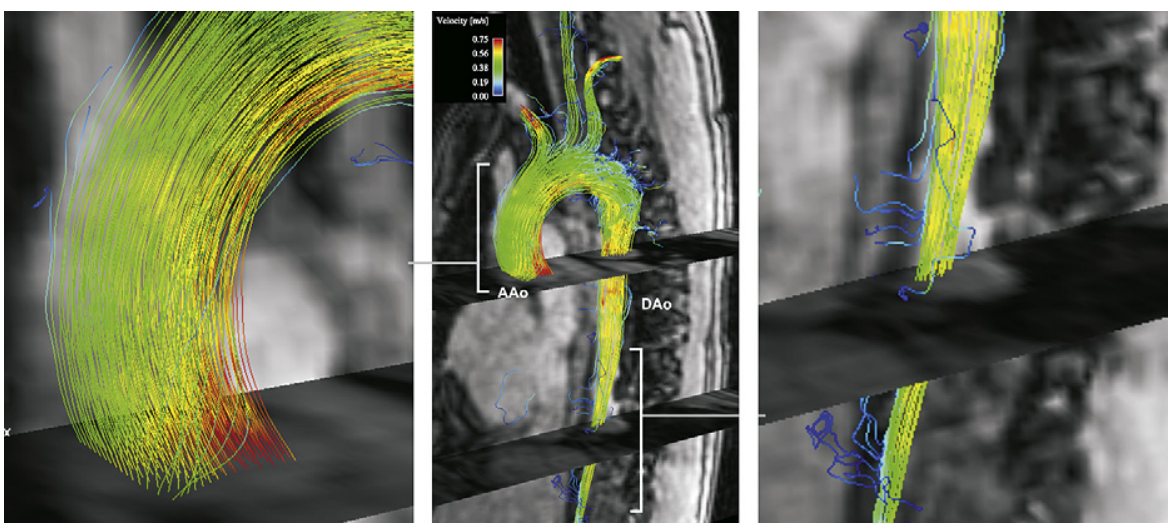

Figure 1. Flow-sensitive 4D MRI at 3T of the aorta and subsequent 3D flow visualization in a healthy volunteer. Measured 3-directional blood flow velocities were visualized using 3D stream-lines depicting systolic flow characteristics in the entire thoracic aorta and supra-aortic branches. Color coding = local velocity magnitude. $A A o$, Ascending aorta; $D A o$, descending aorta.

predominant versus the opposed WSS direction according to $\mathrm{Ku}$ and colleagues ${ }^{17}$ and Moore and colleagues. ${ }^{18}$

The results for all volunteers demonstrated a high interindividual consistency of axial WSS and OSI in the AAo and DAo as indicated by the relatively low standard deviations (Table 1). Although axial WSS was consistently high along the vessel circumference, low OSI values indicate only mild oscillating and thus retrograde flow as expected in young healthy individuals.

\section{Aortic Stenosis}

To illustrate a potential application of the proposed flow measurement and data processing strategy, we also evaluated findings in a 13-year-old boy with a slightly reduced exercise tolerance who presented to the University Hospital Pediatric Cardiology center. Because of constantly higher blood pressures in the upper body (right arm 170/85 mm Hg, left arm $180 / 100 \mathrm{~mm} \mathrm{Hg}$ vs 110/65 mm Hg in both legs), a stenosis was suspected by the referring physician. Cardiac echocardiography including the aortic arch remained unsuspicious with normal diameters of the aortic root, aortic arch, and proximal DAo but a slightly elevated diameter of the AAo $(3.0 \mathrm{~cm})$. During physical examination, decreased pulse waves were palpable at the lower extremities. Auscultation and the rest of the physical examination results were normal. He was referred to a time-resolved 3D CE-MRA. ${ }^{11,12}$

\section{Time-resolved Contrast-enhanced Magnetic} Resonance Angiography Findings

Time-resolved 3D MRA revealed a high-grade stenosis of the DAo (Figure 2). A segment $3.5 \mathrm{~cm}$ in length was reduced in diameter with a maximal reduction of the aortic diameter from $14 \mathrm{~mm}$ to $3 \mathrm{~mm}$. Clearly, a marked dilatation of the internal thoracic arteries and intercostal arteries serving as collaterals can be appreciated.

\section{Hemodynamic Assessment with Flow-sensitized 4-Dimensional Magnetic Resonance Imaging}

To analyze the hemodynamic consequences of the pathology and effects of blood flow alterations on the vessel wall, flowsensitized 4D MRI covering the entire thoracic aorta was performed before stent placement and twice afterwards ( 5 and 9 months after intervention). Subsequent visualization of the measured blood flow velocities and directions included 3D streamline depiction of the flow patterns (Figure 3). The visualization of the stenosis was hampered by the small intrastenotic diameter and aliasing artifacts (Figure 3, A, white arrow). However, 2 main findings were observed: As

TABLE 1. Qualitative image comparison between healthy volunteers and the patient

\begin{tabular}{|c|c|c|c|c|c|c|c|c|c|}
\hline & $\begin{array}{c}\text { Diameter } \\
\text { AAo }\end{array}$ & $\begin{array}{c}\text { No. of } \\
\text { vortices }(n)\end{array}$ & $\begin{array}{c}\text { Vortex } \\
\text { duration }\end{array}$ & $\begin{array}{l}\text { Helical } \\
\text { flow }\end{array}$ & $\begin{array}{l}\text { Retrograde } \\
\text { flow }\end{array}$ & $\begin{array}{c}\text { Mean } \\
\text { WSS AAo } \\
\left(\mathrm{N} / \mathrm{m}^{2}\right)\end{array}$ & $\begin{array}{c}\text { Mean } \\
\text { WSS DAo } \\
\left(\mathrm{N} / \mathrm{m}^{2}\right)\end{array}$ & $\begin{array}{l}\text { OSI } \\
\text { AAo }\end{array}$ & $\begin{array}{l}\text { OSI } \\
\text { DAo }\end{array}$ \\
\hline $\begin{array}{l}\text { Healthy volunteers } \\
\qquad(\mathrm{n}=11)\end{array}$ & $2.4 \pm 0.2 \mathrm{~cm}$ & $\begin{array}{l}0(10) \\
1(1)\end{array}$ & $348 \mathrm{~ms}$ & $\begin{array}{l}\text { Right-handed } \\
\text { (11) }\end{array}$ & $\begin{array}{l}\text { Moderate } \\
\text { (10) } \\
\text { None (1) }\end{array}$ & $\begin{array}{c}0.191 \pm \\
0.06\end{array}$ & $\begin{array}{r}0.158 \pm \\
0.025\end{array}$ & $\begin{array}{r}4.1 \% \pm \\
1.1 \%\end{array}$ & $\begin{array}{r}5.4 \% \pm \\
1.7 \%\end{array}$ \\
\hline Patient & $3.0 \mathrm{~cm}$ & & & & & & & & \\
\hline Before treatment & & 2 & $>338 \mathrm{~ms}^{\mathrm{a}}$ & None & None & 0.140 & 0.158 & 10.2 & 2.5 \\
\hline 5 mo after treatment & & 1 & $>432 \mathrm{~s}^{\mathrm{a}}$ & None & None & 0.127 & 0.237 & 10.8 & 0.0 \\
\hline 9 mo after treatment & & 1 & $365.8 \mathrm{~ms}$ & Right-handed & None & 0.160 & 0.300 & 8.5 & 0.2 \\
\hline
\end{tabular}

$A A o$, Ascending aorta; $D A o$, descending aorta; WSS, wall shear stress; OSI, oscillatory shear index. ${ }^{\text {} V o r t i c e s ~ d i d ~ n o t ~ c e a s e ~ w i t h i n ~ t h e ~ a c q u i r e d ~ t i m e ~ f r a m e s . ~}$ 

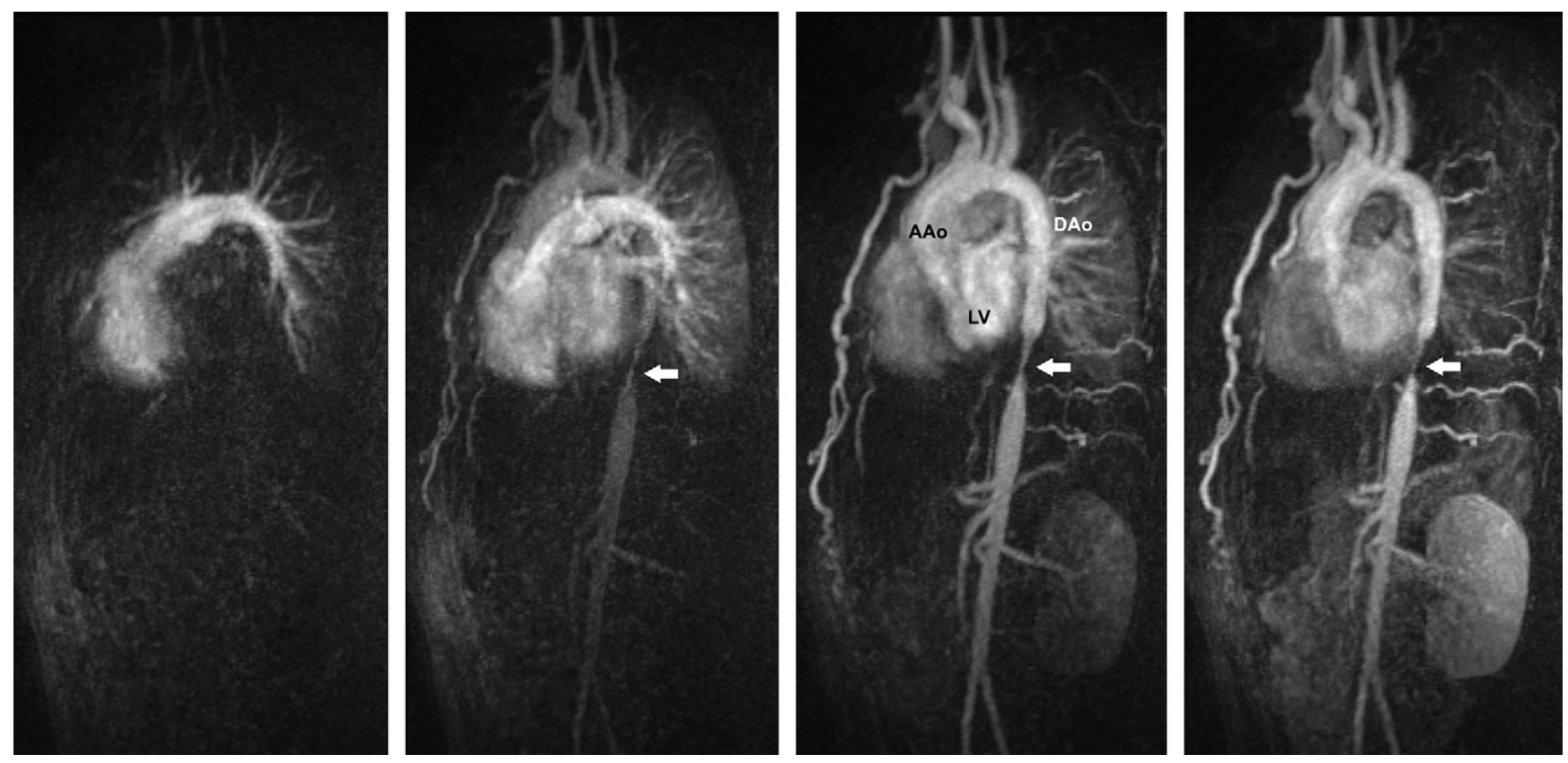

Figure 2. Maximum intensity projections of 4 (of 20) successive time frames representing the first arterial passage of the contrast agent using time-resolved 3D CE-MRA at 3T with a temporal update rate of 2.8 seconds. A high-grade stenosis of the DAo (white arrow) was revealed in this 13-year-old boy. In addition, marked collateral flow via the intercostal arteries and internal thoracic arteries was observed. $A A o$, Ascending aorta; $D A o$, descending aorta; $L V$, left ventricle.

expected, flow distal to the stenosis was accelerated and a backward flow vortex evolved at the posterior part of the vessel (Figure 3, A, right). Even more noticeable, vortical and retrograde flow was seen further upstream in the AAo (Figure 3, A, left). Before intervention, the patient revealed 2 vortices and no helicity of blood flow within the aorta, which was markedly different from findings in healthy volunteers (Table 1). During follow-up, the second vortex vanished after 5 months and the large vortex in the AAo substantially decreased in size (Figure 3,B). Also, blood flow helicity was restored after 9 months showing a right-handed helical movement.

\section{Quantitative Flow Analysis}

Figure 4 compares WSS and flow quantification for planes in the AAo (left) and the proximal DAo (right). Altered hemodynamics led to a vortex formation and locally reversed flow that eventually induced low and oscillating WSS in the thoracic aorta. In comparison with the circumferentially constant high and thus unsuspicious WSS values in the proximal DAo, low WSS values (green bars) with a high OSI (magenta bars, black arrows in Figure 4) were observed at the inner curvature of the AAo. These findings, which can also be seen in older volunteers, ${ }^{9}$ may indicate a vascular area exposed to parameters (low and oscillating WSS) contributing to a vascular remodeling as suggested in the recent litera- ture. ${ }^{2}$ Although there is no direct evidence for the contribution of alterations in WSS and OSI to the development of aneurysm, it may be hypothesized that locally altered hemodynamics and vessel wall parameters, for example, the observed overt difference in values between the anterior and the posterior vessel wall, may affect vascular remodeling and thus aneurysm growth.

Furthermore, the quantitative analysis of blood flow revealed substantial flow reduction distal to the branching supra-aortic vessels, indicating enhanced collateral perfusion as a predictive factor in stenosis. ${ }^{19}$ In addition to a pre-stenotic reduction of the flow waveform in the DAo, a marked reduction in peak flow $(310 \mathrm{~mL} / \mathrm{s}$ vs $60 \mathrm{~mL} / \mathrm{s})$ can be appreciated if the flow curve through the plane transecting the AAo (Figure 4, lower left) is compared with the temporal evolution of flow further downstream in the distal DAo (Figure 4, lower right).

After stenosis repair, there was no change in mean blood flow velocities in the AAo after 5 months but an increase after 9 months (Figure 5, A). In the proximal DAo, blood flow velocities increased significantly and a more physiologic blood flow profile developed. The associated increase in blood flow volume can be explained by a reduction in collateral flow as a consequence of improved flow through the stenosis.

A similar development was seen in the distribution and extent of axial WSS evaluated for 12 segments along the 


\section{A: Systolic 3D stream-lines, entire aorta}
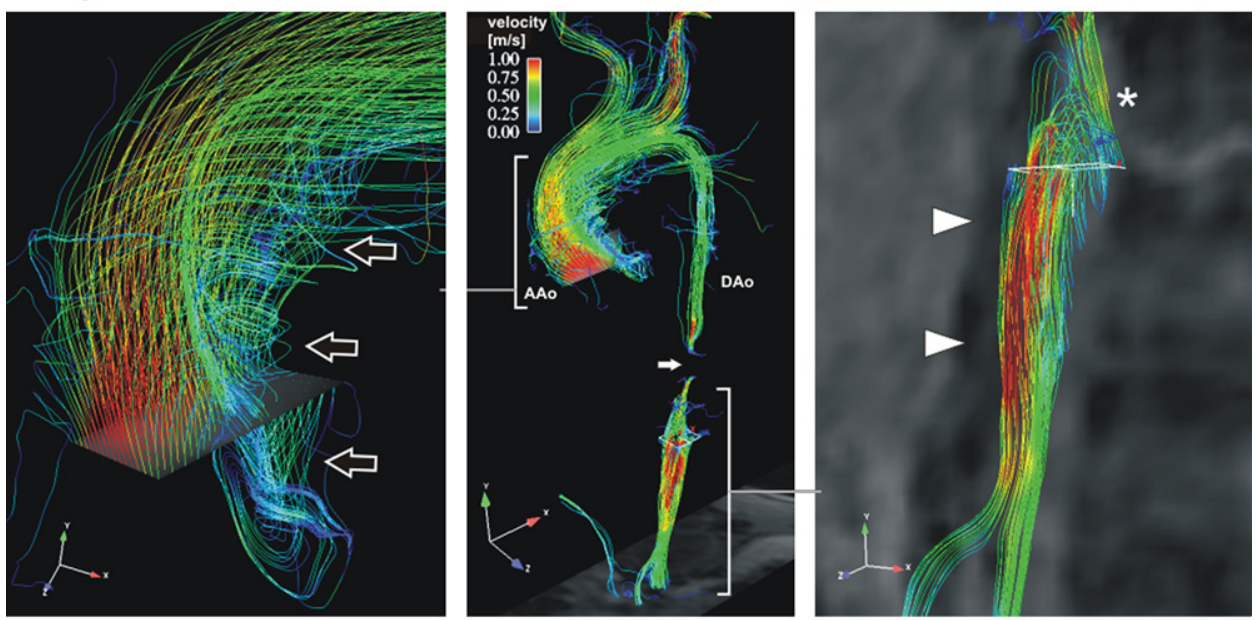

\section{B: Systolic 3D stream-lines in ascending aorta}

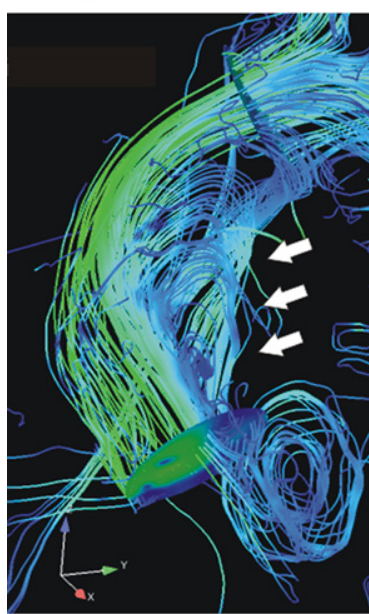

before intervention

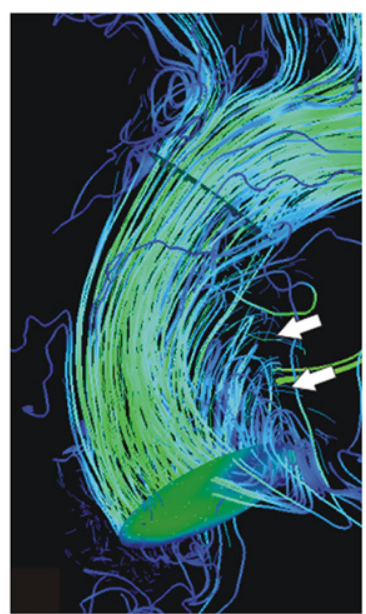

5 months post intervention

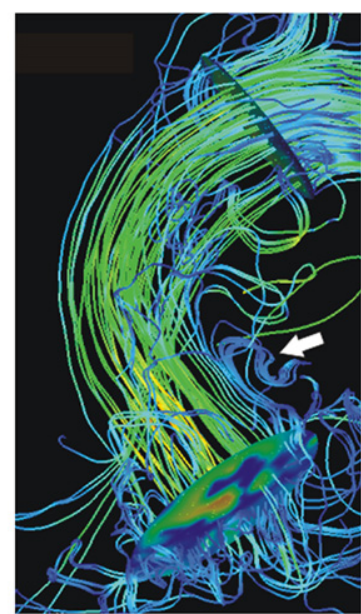

9 months post intervention

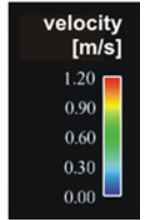

Figure 3. Flow-sensitive 4D MRI at 3T and subsequent visualization using 3D streamlines of the aorta in patient with severe descending aortic stenosis. A, Hemodynamics before intervention included vortical and retrograde flow in the AAo (A left, open white arrows) and accelerated flow distal to the stenosis (A right, white arrowheads) in combination with retrograde flow in the posterior part of the vessel (A right, asterisk). Note that 3D flow visualization was hampered by velocity aliasing and the small diameter of the stenosis (bottom middle, white arrow). B, Systolic 3D streamline visualization of the $A A o$ before $(A)$ and 5 (B) and 9 (C) months after stent placement and dilatation. Note the initially large vortex at the inner curvature of the $A A D$ and proximal arch that decreases after dilatation (solid white arrows). Color coding = local velocity magnitude. 3D, 3-dimensional; $M R I$, magnetic resonance imaging; $A A O$, ascending aorta; $D A o$, descending aorta.

vascular circumference. In both the AAo and DAo, the initial finding of greatly decreased WSS at the inner curvature of the vessel improved. In the proximal DAo, the differences were significant (Figure 5, B).

\section{Discussion}

Although there are other means to diagnose aortic stenosis, the pathologic changes in vascular geometry can be acquired with time-resolved 3D CE-MRA. Moreover, the associated development of collaterals was depicted and led to a balloon angioplasty and endovascular stent placement under neurophysiologic monitoring. The main advantage of time-resolved CE-MRA in children is related to the high temporal update rate of the order of seconds, which allows for an easily performed CE-MRA without the need for contrast agent bolus timing, which can be a comprehensive task. 
WSS vectors $\left[\mathrm{Nm}^{2}\right]$ 12 angular segments
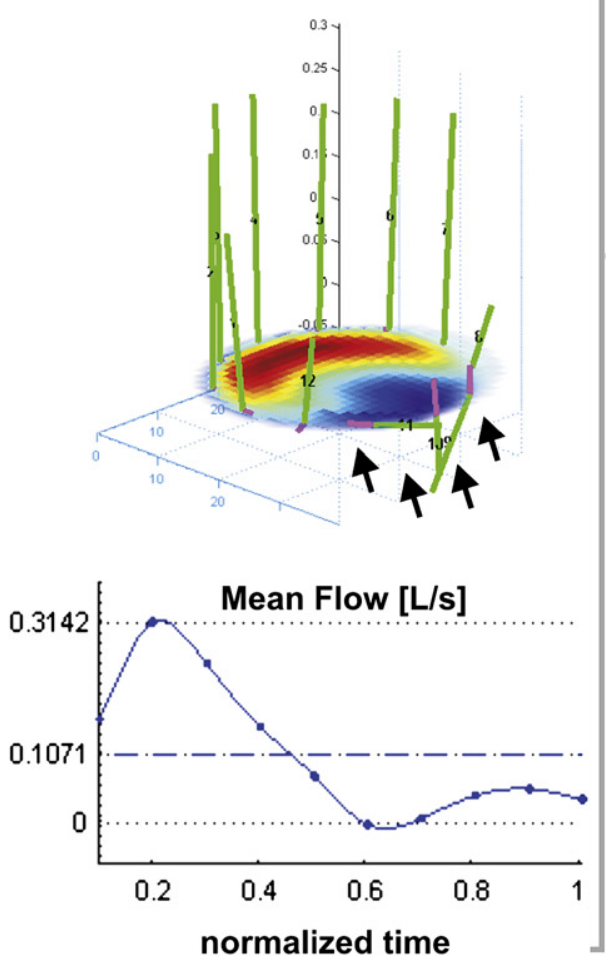
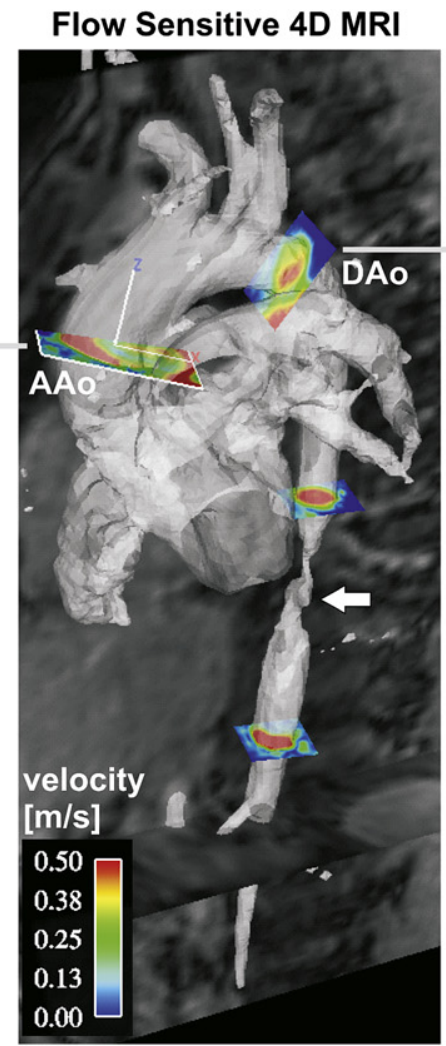

WSS vectors $\left[\mathrm{Nm}^{2}\right]$ 12 angular segments
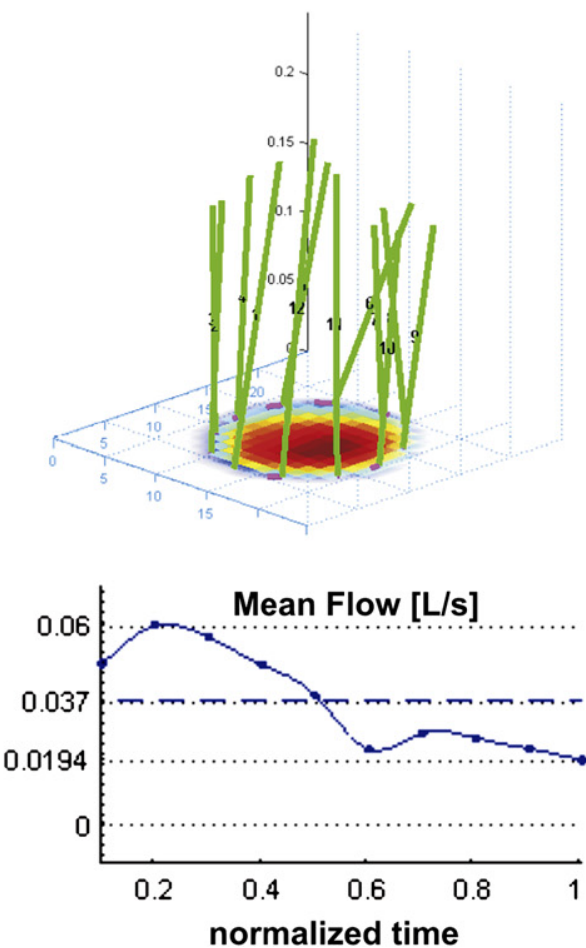

Figure 4. Analysis of WSS and flow quantification: The placement of retrospectively defined 2-dimensional cutplanes transecting the aorta (AAo and DAo) allowed for the quantitative evaluation of blood flow and vessel wall parameters. The selection of cutplanes is illustrated in conjunction with a surface-rendered geometry of the aorta (middle) derived from the same 3D velocity encoded dataset ( $w h i t e$ arrow $=$ stenosis). Altered hemodynamics led to vortex formation and locally reversed flow in the AAo that eventually induced low and oscillating WSS along the inner wall (Figure 3, top left: green bars: mean segmental vectorial WSS $\left[\mathrm{N} / \mathrm{m}^{2}\right.$ ], magenta bars: OSI (multiplied by WSS), bottom: flow quantification showing mean flow in [L/s]) if compared with relatively unsuspicious findings in the proximal DAo (right). The results indicate areas susceptible to vascular remodeling at the inner curvature of the AAo if compared with segmentally more constant WSS with a lower OSI in the proximal DAo. WSS, Wall shear stress; 4D, 4-dimensional; MRI, magnetic resonance imaging; AAo, ascending aorta; $D A o$, descending aorta; OSI, oscillatory shear index.

However, the tradeoff between spatial and temporal resolution can be chosen differently for different imaging demands, for example, if high-resolution morphology is needed and the temporal resolution is secondary. In this case, a temporal update rate of 2.8 seconds and large field of view imaging with an acceptable spatial resolution enabled a detailed analysis of the distal aortic stenosis. ${ }^{12}$

Flow-sensitive 4D MRI in combination with comprehensive visualization and quantification allowed for a characterization of the hemodynamic changes directly associated with the stenosis despite the fact that artifacts limited the direct visualization of the stenosis. In contrast with earlier reports on flow visualization, this analysis at $3 \mathrm{~T}$ is principally possible without the aid of contrast agents and uses a true 3D acquisition instead of a multiple stack approach.
The full 3D coverage of the entire aorta revealed unexpectedly complex vortical and retrograde flow further upstream in the AAo. Detailed quantitative analysis of local flow profiles hinted at considerable collateral flow and revealed noticeably altered WSS at the inner curvature of the AAo. The follow-up examinations focused on hemodynamic analysis of the changes in the AAo and proximal DAo. Timeresolved CE-MRA after stent placement was not conducted because stent placement and signal void did not allow for a diagnostic examination. During analysis, changes in blood flow and WSS were observed that further underline the future possibilities of the methodology and indicate a normalization of hemodynamics after therapy.

These findings implicate a potential change of endothelial function in patients not treated for stenosis even in other parts 

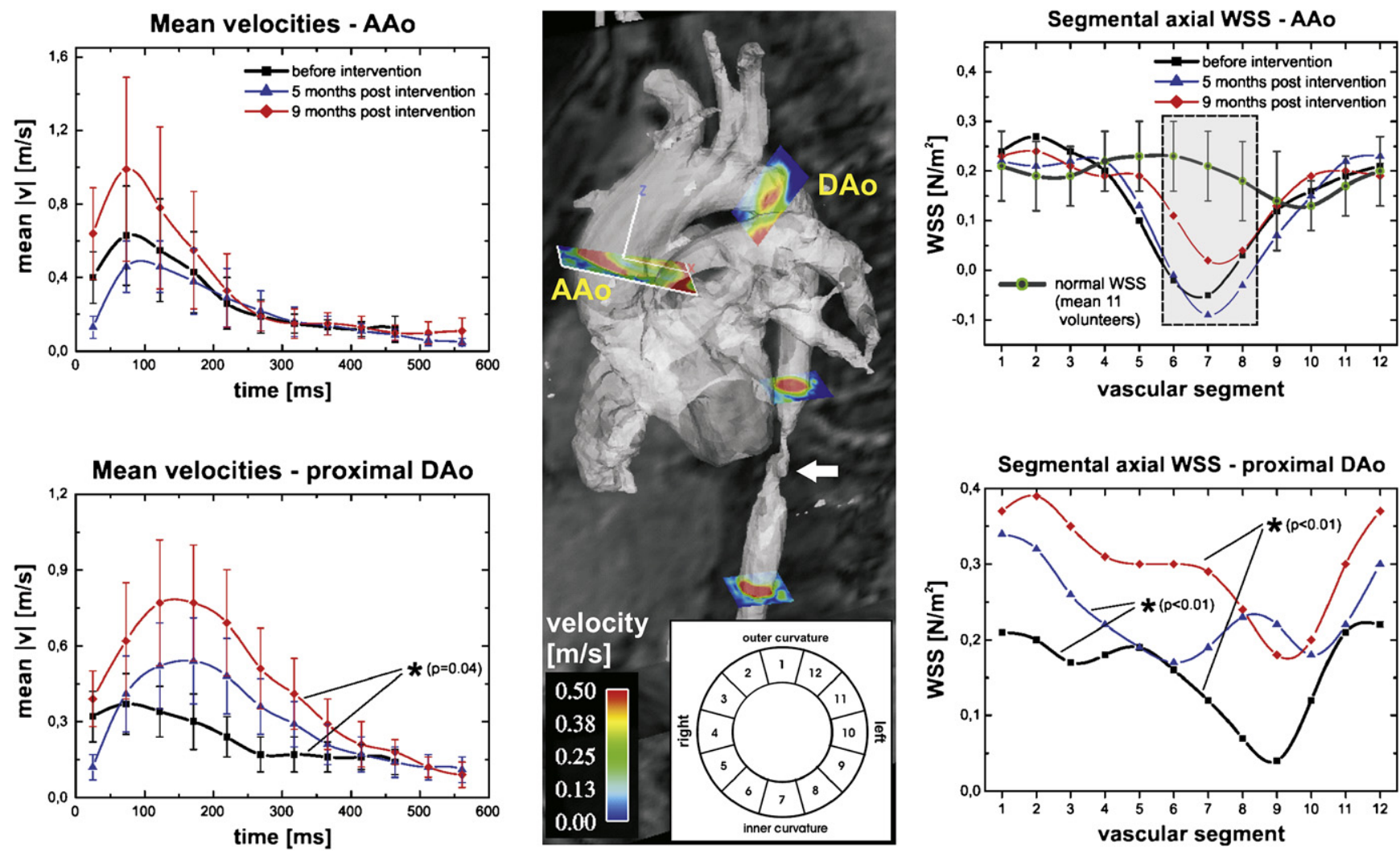

of the circulatory system possibly contributing to aneurysm formation or atherogenesis. Our results therefore contribute to the observation that stenosis can be associated with the evolution of ascending aneurysms, ${ }^{4}$ although further studies with a larger patient collective have to reveal whether these findings can be attributed to endothelial dysfunction or other vascular wall alterations such as fibrosis. Nevertheless, potential clinical implications of hemodynamic changes that can be observed using our novel technology are related to the additional information on regional hemodynamic alterations, which may help to improve therapy management in patients before and after intervention.

The diagnosis and therapy management regarding aortic stenosis is typically made using routine echocardiography or cross-sectional imaging methods. Note that such diagnostic tests provide the correct description of the local pathology (ie, stenosis grade) but may fail to detect secondary complica- tions, such as vortex formation in the AAo, far distant from the pathology as seen in the patient presented in this study.

\section{Conclusions}

The preliminary results demonstrate the potential and additional value of a combined diagnostic imaging strategy with time-resolved 3D CE-MRA and full spatial and temporal coverage of flow-sensitive 4D MRI for the detection of flow alterations and their local impact of the vessel wall far from the original site of the pathology.

\section{References}

1. Wentzel JJ, Corti R, Fayad ZA, Wisdom P, Macaluso F, Winkelman MO, et al. Does shear stress modulate both plaque progression and regression in the thoracic aorta? Human study using serial magnetic resonance imaging. J Am Coll Cardiol. 2005;45:846-54. 
2. Cheng C, Tempel D, van Haperen R, van der Baan A, Grosveld F, Daemen MJ, et al. Atherosclerotic lesion size and vulnerability are determined by patterns of fluid shear stress. Circulation. 2006;113:2744-53.

3. Frydrychowicz A, Harloff A, Jung B, Zaitsev M, Weigang E, Bley TA, et al. Time-resolved, 3-dimensional magnetic resonance flow analysis at $3 \mathrm{~T}$ : visualization of normal and pathological aortic vascular hemodynamics. J Comput Assist Tomogr. 2007;31:9-15.

4. von Kodolitsch Y, Aydin MA, Koschyk DH, Loose R, Schalwat I, Karck M, et al. Predictors of aneurysmal formation after surgical correction of aortic coarctation. J Am Coll Cardiol. 2002;39:617-24.

5. Lotz J, Doker R, Noeske R, Schuttert M, Felix R, Galanski M, et al. In vitro validation of phase-contrast flow measurements at $3 \mathrm{~T}$ in comparison to $1.5 \mathrm{~T}$ : precision, accuracy, and signal-to-noise ratios. J Magn Reson Imaging. 2005;21:604-10.

6. Fink C, Ley S, Kroeker R, Requardt M, Kauczor HU, Bock M. Timeresolved contrast-enhanced three-dimensional magnetic resonance angiography of the chest: combination of parallel imaging with view sharing (TREAT). Invest Radiol. 2005;40:40-8.

7. Griswold MA, Jakob PM, Heidemann RM, Nittka M, Jellus V, Wang J, et al. Generalized autocalibrating partially parallel acquisitions (GRAPPA). Magn Reson Med. 2002;47:1202-10.

8. Hu HH, Madhuranthakam AJ, Kruger DG, Glockner JF, Riederer SJ. Combination of $2 \mathrm{D}$ sensitivity encoding and $2 \mathrm{D}$ partial Fourier techniques for improved acceleration in 3D contrast-enhanced MR angiography. Magn Reson Imaging. 2006;55:16-22.

9. Bogren HG, Buonocore MH. 4D magnetic resonance velocity mapping of blood flow patterns in the aorta in young vs. elderly normal subjects. J Magn Reson Imaging. 1999;10:861-9.

10. Frydrychowicz A, Weigang E, Harloff A, Beyersdorf F, Hennig J, Langer M, et al. Images in cardiovascular medicine. Time-resolved 3-dimensional magnetic resonance velocity mapping at $3 \mathrm{~T}$ reveals drastic changes in flow patterns in a partially thrombosed aortic arch. Circulation. 2006;113:e460-1.
11. Korosec FR, Frayne R, Grist TM, Mistretta CA. Time-resolved contrastenhanced 3D MR angiography. Magn Reson Med. 1996;36:345-51.

12. Frydrychowicz A, Bley T, Harloff A, Jung B, Langer M, Hennig J, et al. Accelerated time-resolved 3D contrast-enhanced MR angiography at 3T: clinical experience in 31 patients. Magn Reson Mat Phys. 2006;19:187-95.

13. Markl M, Draney MT, Hope MD, Levin JM, Chan FP, Alley MT, et al. Time-resolved 3-dimensional velocity mapping in the thoracic aorta: visualization of 3-directional blood flow patterns in healthy volunteers and patients. J Comput Assist Tomogr. 2004;28:459-68.

14. Markl M, Harloff A, Bley TA, Zaitsev M, Jung BA, Weigang E, Langer M, Hennig J, Frydrychowicz A, et al. Time-resolved 3D MR velocity mapping at $3 \mathrm{~T}$ : Improved navigator gated assessment of vascular anatomy and blood flow. J Magn Reson Imaging. 2007;25:824-31.

15. Buonocore MH. Visualizing blood flow patterns using streamlines, arrows, and particle paths. Magn Reson Med. 1998;40:210-26.

16. Stalder AF, Frydrychowicz A, Canstein C, Hennig J, Markl M. Quantitative planar analysis of flow sensitive 3D CINE MRI. In Proc: ISMRM Workshop on Flow and Motion: Imaging Assessment of Cardiovascular and Tissue Mechanics, New York, NY, USA, June 2006.

17. Ku DN, Giddens DP, Zarins CK, Glagov S. Pulsatile flow and atherosclerosis in the human carotid bifurcation. Positive correlation between plaque location and low oscillating shear stress. Arteriosclerosis. 1985; 5:293-302.

18. Moore JE Jr, Xu C, Glagov S, Zarins CK, Ku DN. Fluid wall shear stress measurements in a model of the human abdominal aorta: oscillatory behavior and relationship to atherosclerosis. Atherosclerosis. 1994;110: 225-40.

19. Steffens JC, Bourne MW, Sakuma H, O'Sullivan M, Higgins CB. Quantification of collateral blood flow in coarctation of the aorta by velocity encoded cine magnetic resonance imaging. Circulation. 1994;90:937-43. 